\title{
Effect of Increasing Number of Nodes on Performance of SMAC, CSMA/CA and TDMA in MANETs
}

\author{
Samiullah Khan, Farooq Faisal, \\ Mahmood Nawaz, Farkhanda Javed \\ Institute of Business Management Sciences \\ The University of Agriculture Peshawar \\ Peshawar-Pakistan
}

Fawad Ali Khan, Rafidah MD Noor Department of Computer System and Technology University of Malaya, Kuala Lumpur Malaysia

\author{
Matiullah, Zia ullah, Muhammad Shoaib, Faqir Usman Masood \\ Department of Basic Sciences and Islamiat \\ Department of Computer System Engineering \\ University of Engineering and Technology \\ Computer science Department, Qurtuba University \\ Peshawar-Pakistan
}

\begin{abstract}
The importance of Wireless Sensor Network (WSN) increases due to deployment for geographical, environmental and surveillance purpose in war fields. WSN facing several challenges due to its complex nature including key problems, such as routing and medium access control protocols. Several approaches were proposed for the performance evaluation of WSN on the basis of these issues due to the fact that MAC layer access protocols have a great impact on the performance of WSN. In this paper, we investigated the performance evaluation of three well known MAC Access protocols, i.e. sensor medium access control protocol (SMAC), carrier sense multiple access with collision avoidance (CSMA/CA), and time division multiple access (TDMA) over adhoc on demand distance vector (AODV) routing protocol. The number of simulation scenarios were carried out by using NS2, the simulation metrics used are throughput, end-to-end delay and energy consumed. Simulation results showed that SMAC out perform CSMA/CA and TDMA by consuming less energy, less end to end delay and high throughput due to contention based approach to access the medium for transmission.
\end{abstract}

Keywords-Medium access control (MAC); sensor medium access control (SMAC); time division multiple access (TDMA); carrier sense multiple access with collision avoidance (CSMA/CA); ad-hoc on demand distance vector (AODV)

\section{INTRODUCTION}

The ad-hoc network is a network of self configuring mobile nodes that can communicate with each other without any centralized control. Intermediate nodes act as a router to forward data from source to destination. In ad-hoc networks, the nodes can move freely and the topology may change frequently due to nodes mobility. Every node in this network acts as host and router. Intermediate nodes act as a router to forward data from source to destination. Routing protocols are responsible for keeping track of the paths from source to destination in their routing tables. Routing tables are constructed by exchanging control messages to keep the information updated [1]. These messages causes extra overhead of the routing protocol and degrades the performance. An efficient routing protocol utilizes the network resources by minimizing delay and power consumption to provide better QOS and maximizing throughput, remains a challenging issue for the ad-hoc networks. Energy consumption is a major factor and have a great impact on the network performance in battery driven nodes in these networks [16].

Medium access control (MAC) plays an important role in the successful operation of the network. One fundamental task of MAC is to avoid collision, so that nodes in the same interference range do not transmit at the same time. The two main operations performed at medium access layer are controlling when to send and when to listen for a packet. There are many MAC protocols that are developed for wireless networks.Typical examples are TDMA, CDMA, CSMA/CA, SMAC, etc. The motivation for the selection of TDMA, CSMA/CA and SMAC in this research work were due to diverse type of access mechanism used by these protocols and have potential for further improvement in order to conserve the battery energy as mentioned in related work section [17]. The rest of the paper is organized as follows. Section II presents the overview of Medium Access protocols and the Routing protocol used and Section III describes the related work done in literature. Simulation environment and results are shown in Section IV and the paper is concluded in Section V.

\section{A. Medium Access Control (MAC) Protocols}

S-MAC uses three novel techniques to reduce energy consumption and support self-configuration as shown in Fig. 1. Nodes periodically sleeps by entering from listening to the idle channel to reduce energy consumption. During transmission of other nodes, SMAC sets the radio to sleep and it only uses in-channel signaling. An application that requires a store-andforward processing as data move through the network message passing are applied by SMAC to reduce contentious latency of the network [18]. 


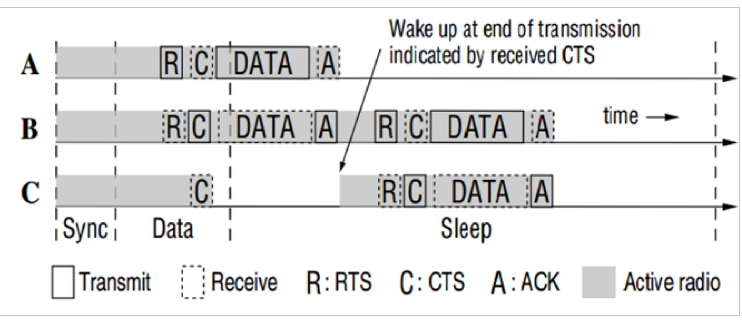

Fig. 1. S-MAC with adoptive listening [1].

CSMA/CA works on the principle of sensing before sending as shown in Fig. 2. As soon as a node receives a packet, it sense the medium and try to send it if the medium is clear or wait a randomly chosen period of time (2 RTT) if another transmission is going on and then checks again. This waiting time period is called back-off factor and is counted down by a back-off counter. If the back-off counter reaches zero and the channel is CSMA/CA will send the packets otherwise the back-off factor is set again and the process will be repeated [2].

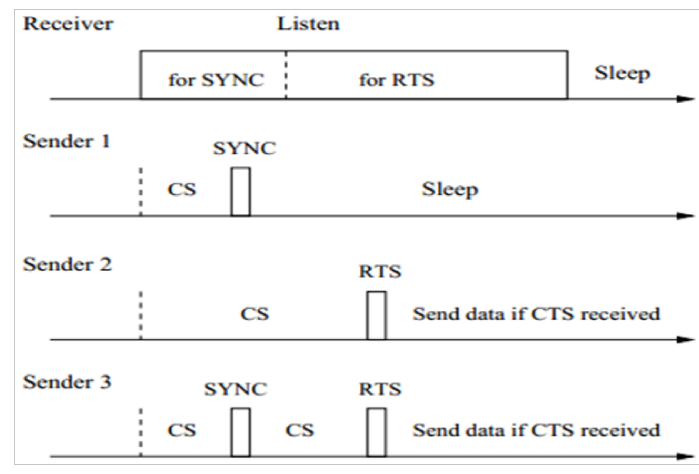

Fig. 2. Timing relationship between receiver and different senders [3].

TDMA shares a single carrier with several users to make use of non-overlapping time slots for each user as shown in Fig. 3. In TDMA, transmission of data is not continuous but occurs in bursts which results in low consumption of energy. For transmission and reception different, the time slots are used by TDMA, thus duplexes are not required. Bandwidth can be supplied on demand to different users by concatenating or reassigning of time slots based on priority.

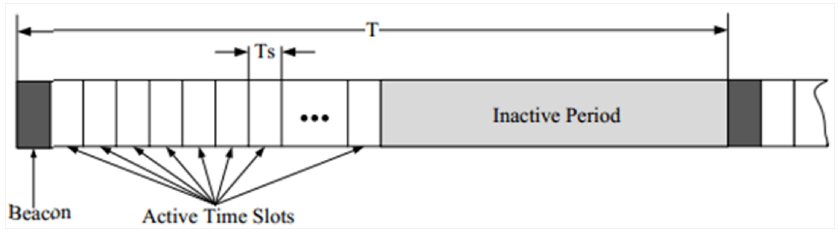

Fig. 3. TDMA frame structure [4].

This paper also investigates the behavior of the popular reactive routing protocol AODV over varying MAC protocols.

\section{B. Routing in Ad-hoc Networks}

Routing is the process of finding the shortest and optimal paths from source to destination in any network. Routing protocols for ad-hoc network can be classified into three main categories, i.e. Reactive, proactive and hybrid. Reactive routing protocols are more popular in ad-hoc networks because they are scalable and creating less overhead. In reactive routing protocols, The routes are established at the time when a node wants to communicate with another node in the network. There are no predefined route which results in reduction of overhead due to the fact that there is no exchange of information to keep track of the network topology [19].

Adhoc On-Demand Distance Vector (AODV) is a reactive routing protocol and works on an on-demand basis, i.e. a route is established only when it is desired by a source node. Routing table is maintained for every node in which the required information for communicating with other users in the network are stored. It reacts to the changes by maintaining only the active routes in the tables for a specified amount of expiration time [20]. AODV identify the most recent path by employing destination sequence numbers. The routes in AODV are expected to be available at a given instant. AODV uses three types of messages to be exchanged for establishing and maintaining routes i.e. route request (RREQ), route reply (RREP) and route error (RERR). When a node wants to communicate, it sends the RREQ as broadcast to its neighbor nodes. The node with the destination address will reply by sending RREP as unicast to the desired source node. It may be possible that a node loses connectivity due to some reason. The next hop nodes will notify all the nodes by sending RERR messages, to keep the information updated [5]. Due to this reactive approach, AODV was used as routing protocol in this study.

\section{RELATED WORK}

Aggarwal et al. (2012) worked on the security challenges faced by ad-hoc routing protocols and proposed (AODVSEC) by enhancing the scope of AODV for the provision of security and compared it with (AODV) and SAODV in normal situation as well as in the presence of three concerned attacks i.e Resource Consumption (RC) attack, Route Disturb (RD) attack, Route Invasion (RI) attack and Black hole (BH) attack. The proposed scheme performed better than AODV and similar to SAODV but with less processing requirements which leads to save a lot of computational power [6].

Chen et al. (2012) studied the routing protocol for low power and lossy network (RPL) and the challenges faced by the protocol to find effective approaches to simulate and emulate the behavior and other extensions of applications. Their work has first provided a brief detail of the RPL protocol by considering two case studies (Contiki RPL and Tiny RPL) and initial experimental simulation results obtained from the COOJA simulator which is RPL capable. Secondly, their work was focused on the utilization of the RPL protocol in the agriculture sector by proposing dedicated hybrid network architecture [7].

Garg and Gupta (2012) studied the behavior of AODV routing protocols and highlighted the dynamic nature of MANETs such as limited power of nodes, bandwidth constrained, variable wireless links and dynamic topology that leads to performance degradation. They implement AODV protocol for optimizing the routing by reducing the loss rate in AODV. 
NS-2 is used for simulating scenarios such as variation in the speed of nodes, packet transfer rates and number of nodes. this work concluded that AODV increases network performance by increasing the packet delivery ratio and reduced network delay [8].

Updhayayet et al. (2012) investigated the behavior of routing in ad-hoc network and the problem faced by the routing protocols by testing both reactive and pro-active protocols for efficiency under different circumstances. They first increase the density of nodes for analyzing the efficiency of the protocols. The metric used were packet delivery ratio, packet loss and end-to-end delay. The simulation showed that reactive routing protocol performed well in these circumstances as compared to proactive [9].

Taneja et al. (2010) reported the experimental analysis of the two prominent on-demand routing protocols by presenting their functionality with varying number of nodes. An effort was made by the author to perform analysis of a new random way point self created network scenarios. The performance parameter taken was the packet delivery ratio in the network scenario of changing speed of the nodes. The results reflected in graphs showed that both protocols are best in its own way, but AODV was emphasized best on the basis of well performing in dense condition [10]

Van Hoese et al. (2004) studied EMAC, a medium access protocol designed for WSN. The EMAC work on top of the effective TDMA scheme, in which each active node listens to the channel periodically and broadcast control messages for sending various types of information by utilizing low energy. The active node performs normal network functions while the passive nodes set aside to save energy. There are two operational modes, the monitoring mode in which the network merely keeps the network connected in a low duty cycle and communication mode in which the nodes are active and transfer data. Their simulation results showed that the presented approach is better as opposed to SMAC protocol in terms of power consumption [11].

Hang Su et al. (2009) proposed a cross layer based batteryaware medium access protocol based on TDMA for monitoring of body area network in wireless health care application. Their proposed approach provides a solution for electrochemical properties of the battery, the time varying wireless fading channels, and packet queuing by prolonged lifespan of battery with reliable and timely guaranteeing delivery of messages in WSN, which is the typical requirement of the patient monitoring network. Analytical and simulation results showed their proposed work performed better by consuming less energy as compared to the IEEE 802.15.4 and Bluetooth protocols [12].

Giuseppe Bianchi et al. (2001) studied the effect of medium access protocol on the performance of WSN by considering different MAC protocols. CSMA/CA is one of the medium access protocol for wireless sensor networks which decreases throughput as the number of nodes in the network increases, the throughput is much better in CSMA/CA with RTS/CTS. In these simulation scenarios, CSMA/CA was compared with TDMA and SMAC by analyzing the throughput, end to end delay and energy consumption [13].

Wei Y et al. (2002) presented a new type of mac protocol designed especially for wireless sensor networks. Energy conservation and self configuration of nodes is the primary goal of the protocol, while latency and per node fairness is less important. SMAC used three novel techniques to consume less energy and support for self configuration. To consume less amount of energy, nodes periodically sleeps to move from listening to idle state. Further, they reduced contention latency for sensor network application by message passing technique that required store-and-forward processing as data move across the network. The simulation showed that SMAC consumes 2 to 6 times less energy as opposed 802.11 like for traffic load with a message sent every $1-10$ secs.

Demirkol et al. (2006) studied various aspects of the WSN to be kept in mind while developing a MAC layer protocol. Target detection, temperature judgment and hospitals are some of the areas in which different application of WSN is applicable and therefore hot areas for researchers [21]. MAC access protocols are the best choice for utilization of energy resources in WSN. A comparison of two MAC access protocol is made by considering the metric as throughput, delay and energy consumed. The simulation results show that both the protocol performed well in different condition and SMAC consumes less energy as compared to TDMA [14].

Anis Koubaa et al. (2006) showed the performance limitation of slotted CSMA/CA mechanism of sensor nodes by enabling beacon mode for broadcast transmission in WSNs. The work was conducted by modelling the CSMA/CA mechanism on top of realistic physical layer with respect to IEEE 802.15.4 standard specification. The parameter used for analysis are throughput and delay. The simulation was conducted using NS-2 by enabling beacon mode in the first case and disabling beacon mode in the second case. Beacon enabled mode showed efficient and good results as compared with the non-beacon mode, which provide synchronization and contention free period [15].

\section{RESULT AND DISCUSSION}

This paper investigates the performance evaluation of Medium access protocol, i.e. SMAC, CSMA/CA and TDMA over AODV routing protocol. The simulation has been performed in the Network Simulator-2 (NS-2), which provides a scalable environment for wireless sensor networks. The simulation area was $2000 \mathrm{X} 2000 \mathrm{~m}$ square and the number of nodes used was 5, 10, and 20 and each node have 1000 joule of initial energy. The Constant Bit Rate (CBR) was used as the application layer traffic for all scenarios with an interval time of 1,5 , and 10 Seconds. The packet size, transport and routing protocol were 50 bytes, User Datagram protocol (UDP) and AODV, respectively. The simulation time was kept 1000 seconds. The configuration of simulation parameters are mentioned in Table I.

The simulation was performed using NS-2 which provides a scalable environment for WSNs. A number of simulation scenarios (Scalability and interval) were carried out by using performance analysis parameters i.e. throughput, end to end delay and energy consumed. The scalability scenario was designed with motivation to find out the performance efficiency of MAC layer protocols with increasing number of nodes various sub scenarios. The number of nodes used for scalability 
TABLE I. General Simulation Parameters Configurations

\begin{tabular}{|c|c|c|}
\hline S/No & Attributes & Values \\
\hline 1 & Simulator & NS-2 (Version 2.34) \\
\hline 2 & Application Layer Protocol & Constant Bit Rate(CBR) \\
\hline 3 & MAC Layer Protocol & SMAC, CSMA/CA and TDMA \\
\hline 4 & Nodes Intial Energy Leve & 1000 Joules \\
\hline 5 & Packet Size & 50 Bytes \\
\hline 6 & Number of Nodes & 5,10 and 20 Nodes \\
\hline 7 & Routing Protocol & AODV \\
\hline 8 & Simulation Time & 1000 Seconds \\
\hline 9 & Area (m2) & $2000 \times 2000$ \\
\hline 10 & Traffic Type & CBR/UDP \\
\hline 11 & Data Interval & 1,5 and 10 mseconds \\
\hline
\end{tabular}

scenarios were 5, 10, and 20. The Interval scenarios described real behavior of the nodes in the WSNs in which do not communicates continuously. To simulate this behavior, three Interval scenarios were designed with an interval of 1,5 and $10 \mathrm{~ms}$.

\section{A. Scalability Scenario}

The SMAC has high throughput in all three sub scalability scenarios as compare to the CSMA/CA and TDMA. The CSMA/CA is a contention base protocol in which the number of collision increases as the number of nodes in the network increased. TDMA is conflict free protocol and contention is avoided by the use of reserving the slot for each transmitter. SMAC has comparatively higher throughput as shown in Fig. 4. SMAC has features of contention and scheduling. Throughput of SMAC rises with the increase in node density.

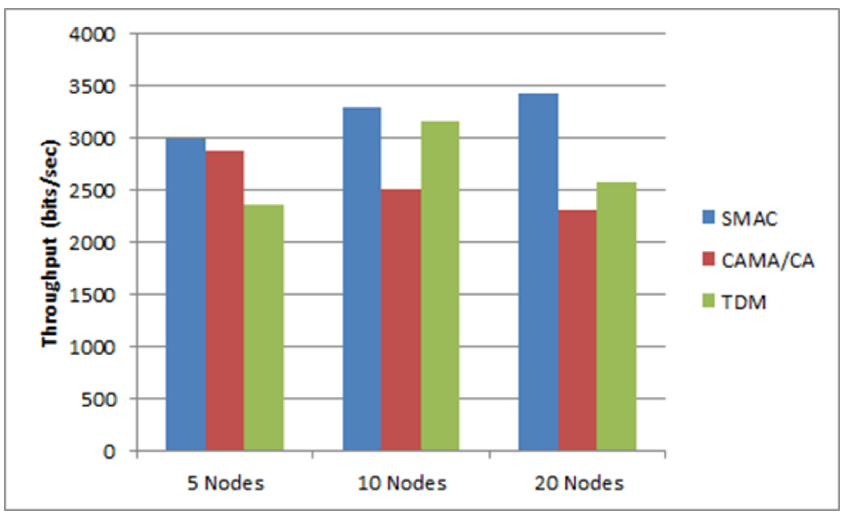

Fig. 4. Throughput of SMAC, CSMA/CA and TDMA in scalability scenario.

CSMA/CA has a comparatively higher delay (as shown in Fig. 5) with increase in the number of nodes in the network. This increase in delay of CSMA/CA is due to collisions and set back off the counter. This result in the comparatively less end to end delays, with the increase in the time interval. The simulation graph gives a clear picture of SMAC that have comparatively much lower delay as opposed to the other two MAC protocol.

The energy consumption of SMAC protocol is comparatively much lower than CSMA/CA and TDMA as shown in Fig. 6. SMAC used predictive techniques where nodes goes to idle state from communicating state due to periodic sleep.

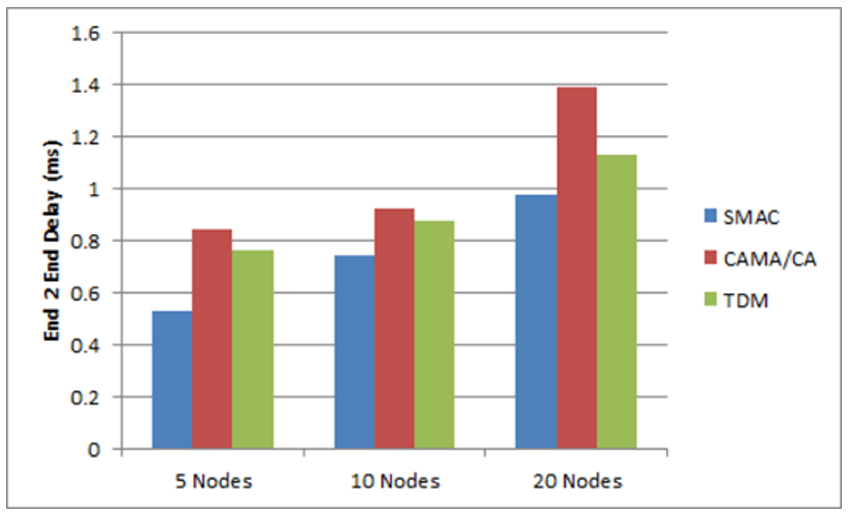

Fig. 5. End-2-end delay of SMAC, CSMA/CA and TDMA in scalability scenario.

This helps in reducing energy consumption of the protocol. The CSMA/CA senses the medium all the time for transmission and reception of data which increased the energy consumption of the protocol. Moreover, there is no predictive techniques used in TDMA which also consumes higher energy as compared to SMAC.

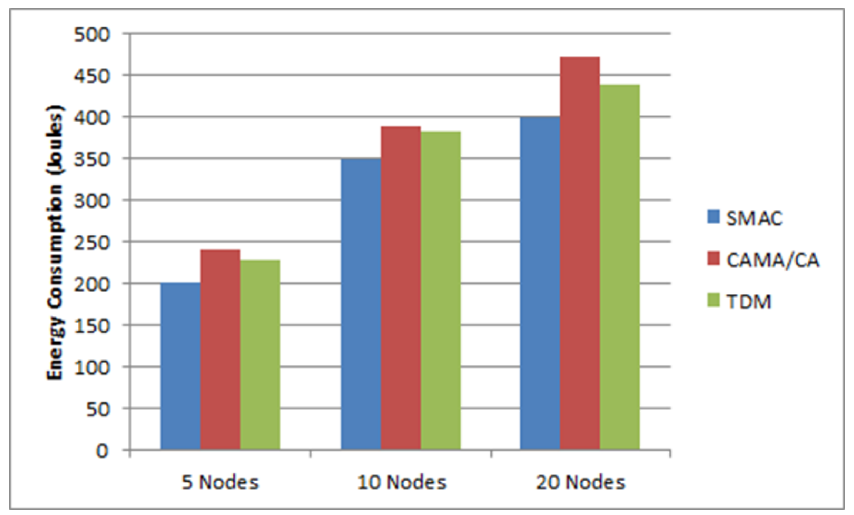

Fig. 6. Energy consumption of SMAC, CSMA/CA and TDMA in scalability scenario.

\section{B. Interval or Data Rate Scenario}

The number of nodes in interval scenarios are 20 and the interval time was kept 1,5 , and $10 \mathrm{~ms}$. The throughput of all three MAC layer protocol decreases as the time interval increases as shown in Fig. 7. SMAC showed comparatively higher throughput as opposed to CSMA/CA and TDMA as shown in figure. This is due to the periodic sleeping techniques used by Sensor Medium Access protocol.

CSMA/CA and TDMA has comparatively higher delay as compared to SMAC as shown in Fig. 8. The simulation results showed that SMAC performed well and reduced the delay even when the time of interval was further increased. $1 \mathrm{~ms}$ interval corresponding to higher traffic while traffic generation decreased as the time interval was increased in the network.

The power consumption of TDMA and CSMA/CA is much higher than SMAC medium access protocol as shown in Fig. 9. The sleep delay prediction of SMAC gives the ability to protocol to consume less energy while TDMA and 


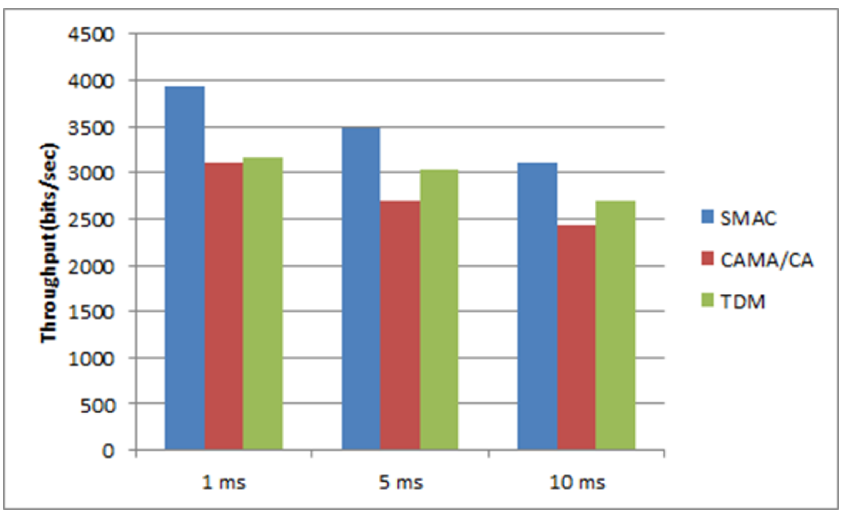

Fig. 7. Throughput of SMAC, CSMA/CA and TDMA in interval scenario.

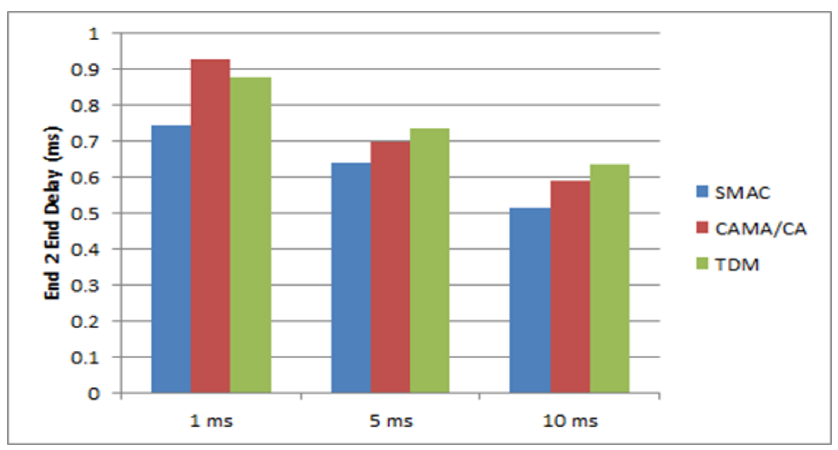

Fig. 8. End 2 end delay of SMAC, CSMA/CA and TDMA in interval scenario.

CSMA/CA was listening continuously to the medium which results in high consumption of energy.

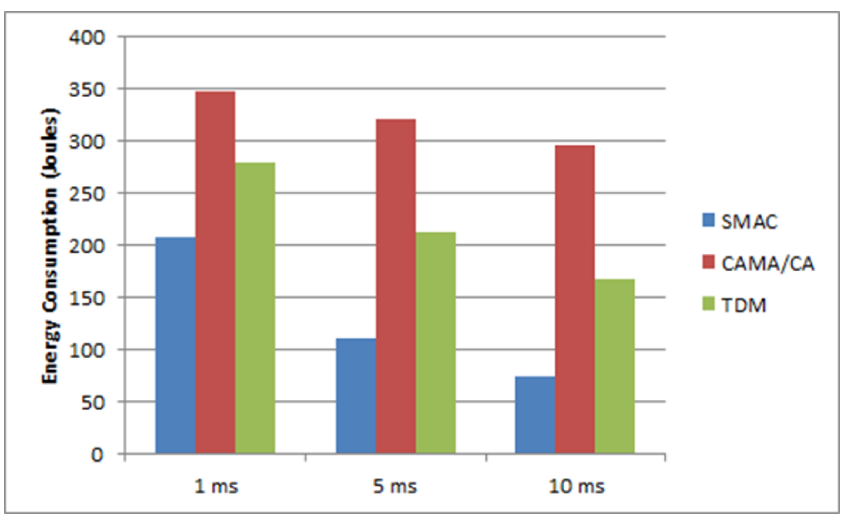

Fig. 9. Energy consumption of SMAC, CSMA/CA and TDMA in interval scenario.

\section{CONCLUSiON}

This paper examines the performance evaluation of three Medium Access Protocols, i.e. SMAC, TDMA and CSMA/CA over the AODV routing protocol for Wireless Sensor Networks (WSNs). The simulation results conclude that SMAC perform much better in both scalability and time interval scenarios as compared to the other medium access protocols, i.e. TDMA and CSMA/CA. The CSMA used contention to find out the collision by continually sensing the medium until getting free transmission medium. The conflict free MAC layer protocol, i.e. TDMA helped the nodes in avoiding the overlapping during transmission in WSNs. The SMAC was hybrid in nature as it have adopted the both the contention based mechanism of CSMA/CA to get the medium for transmission and conflict free nature of TDMA for dynamic allocation of medium or resources. It is concluded from the study and simulation results that SMAC performs better on AODV in WSNs in the given circumstances. Further research can be done in the same area by considering different simulation scenarios such as using different traffic pattern and routing protocols.

\section{REFERENCES}

[1] A. Aggarwal and B. Garg (2012), Survey on secure aodv for ad-hoc networks routing mechanism.Int J Adv Res Comput Sci Softw Eng, vol. 2, no. 3 , pp. 203-206.

[2] H. S. Bindra, S. K. Maakar, and A. Sangal (2010), Performance evaluation of two reactive routing protocols of manet using group mobility model. International Journal of Computer Science, vol. 7, no. 3, pp. 38-43.

[3] E. Ziouva and T. Antonakopoulos(2002) Csma/ca performance under high traffic conditions: throughput and delay analysis Computer communications, vol. 25, no. 3, pp. 313-321.

[4] B.-C. Kim (2001) Method for forming frame structure for use in time division multiple access communication system. uS Patent 6, pp.172-971.

[5] M. Alshowkan, E. A. Fattah, and A. Odeh,(2012) Performance evaluation of dymo, aodv and dsr routing protocols in manet. International Journal of Computer Applications, vol. 49, no. 11. pp. 176-191.

[6] A. Aggarwal, S. Gandhi, N. Chaubey, P. Shah, and M. Sadhwani (2012) Aodvsec: A novel approach to secure ad hoc on-demand distance vector $(a o d v)$ routing protocol from insider attacks in manets. arXiv preprint arXiv: 1208.1959

[7] Y. Chen, J.-P. Chanet, and K. M. Hou (2012) Rpl routing protocol a case study: Precision agriculture. First China-France Workshop on Future Computing Technology, pp. 6-17.

[8] A. Lal, S. Dubey, and B. Presswani (2012) Reliability of manet through the performance evaluation of aodv, $d s d v, d s r$. International Journal of Advanced Research in Computer Science and Software Engineering, vol. 2 , no. 5 , pp. 125-134.

[9] S. Upadhyay, P. Joshi, N. Gandotra, and A. Kumari (2012) Comparison and performance analysis of reactive type $d s r$, aodv and proactive type $d s d v$ routing protocol for wireless mobile ad-hoc networking, using ns2 simulator. Journal of Engineering and Computer Innovations. vol. 2, no. 10 , pp. $36-47$.

[10] S. Taneja and A. Kush (2010) A survey of routing protocols in mobile ad hoc networks. International Journal of Innovation, Management and Technology, vol. 1, no. 3, pp. 279-291.

[11] L. Van Hoesel and P. J. Havinga (2004) A tdma-based mac protocol for wsns in Proceedings of the 2nd international conference on Embedded networked sensor systems. pp.303-304.

[12] H. Su and X. Zhang (2009) Battery-dynamics driven tdma mac protocols for wireless body-area monitoring networks in healthcare applications. Selected Areas in Communications, vol. 27, no. 4, pp. 424-434.

[13] Y. Tay and K. C. Chua (2001) A capacity analysis for the ieee 802.11 mac protocol. Wireless networks, vol. 7, no. 2, pp. 159-171.

[14] I. Demirkol, C. Ersoy, F. Alagoz (2006) Mac protocols for wireless sensor networks: a survey IEEE Communications Magazine, vol. 44, no. 4, pp. 115-121.

[15] A. Koubaa, M. Alves, and E. Tovar (2006) A comprehensive simulation study of slotted csma/ca for ieee 802.15. 4 wireless sensor networks in 5th IEEE International Workshop on Factory Communication Systems.pp. 183-192.

[16] Khan, S. and Qadir, M. A. (2015). Inter-path OOS packets differentiation based congestion control for simultaneous multipath transmission. Int. Arab J. Inf. Technol. Vol.4, No.6, pp.907-913. 
[17] Khan, S. and Qadir, M. A. (2017). Deterministic Time Markov Chain Modelling of Simultaneous Multipath Transmission Schemes. IEEE Access, Vol. 5, pp.8536-8544.

[18] Ali, H., Khan, S. and Quaid, M. (2015). Comparative analysis of controlled delay (CoDel) with Deficit Round Robin (DRR) to overcome bufferbloat problem in wired network. International Journal of Current Engineering and Technology, Vol.5, No. 5, pp. 3378-3386.

[19] Khan, F., Abbas, S. and Khan, S. (2016). An Efficient and Reliable Core-Assisted Multicast Routing Protocol in Mobile Ad-Hoc Network.
International journal of advanced computer science and applications, Vol. 7, No. 5, pp. 231-242.

[20] Shakir, S. Khan,S. Hassain, L. and Matiullah (2017) QoS Based Evaluation of Multipath Routing Protocols in Manets, Advances in Networks. Vol. 5, No. 2, 2017, pp. 47-53. doi: 10.11648/j.net.20170502.13

[21] Khan, F., Abbas, S. and Khan, S. (2018). Secure Core-Assisted Multicast Routing Protocol in Mobile Ad-Hoc Network, Journal of Internet Technology, Article In Press. 